\title{
MAINTENANCE CONTRACT MODEL FOR COMPLEX ASSET/EQUIPMENT
}

\author{
Anisur Rahman \\ Griffith School of Engineering, Griffith University, Australia \\ a.rahman@griffith.edu.au
}

\begin{abstract}
Maintenance contracts received significant attentions in the past fifteen-twenty years as it has huge potential to reduce the upfront investments in maintenance infrastructure, specialised maintenance facilities, and risks to the owners through expert services provided by the original equipment manufacturers and/ or specialist maintenance providers. To make maintenance contracts more effective, there is a need to develop mathematical models and understand future costs that could be built into the contract price. In this paper, a conceptual model is developed for estimating cost of outsourcing maintenance of complex and critical asset/equipment taking into account both corrective and preventive maintenance as servicing strategies and risks and penalty costs associated with such contract. The developed cost models are analysed using illustrated numerical examples.
\end{abstract}

Keywords: Maintenance Contract, Rectification, Cost Model

\section{Introduction}

For expensive and complex assets/equipment (e.g. power generation plants, rail networks etc), the maintenance services need to have expertise, and specialized facilities and infrastructure. Often it is found to be expensive for the owner of such asset/equipment to have well-built infrastructure, specialized maintenance facilities and specialists in house. This has resulted in a growing trend for the owners of asset intensive industries to outsource the management of maintenance activities of their complex assets to external agencies. Outsourcing through maintenance contracts are becoming more and more popular to the owners of such items as maintenance through contracts has potentiality to reduce upfront investments in infrastructure, expertise and specialized maintenance facilities ${ }^{1,6}$. On the other hand, maintenance contracts have received significant attention to the service agents/providers due to increased profits and reduction of risks through expert services. A maintenance contract is the outsourcing of maintenance actions where defects/failures are rectified by an external agent (service provider) for an agreed period of time. The agent in turns charges a price for such service. The service providers can be one of their asset operators or manufacturers of the asset or independent third parties, interested in investing for asset infrastructures ${ }^{7}$. As a result, maintenance contracts have become a billion dollars annual business. Typically, at least half of that goes into the service provider's pocket as profit, with less than $20 \%$ spent on the repair or replacement of item ${ }^{10}$. Therefore, there is a great need for cost estimation of maintenance contracts in order to balance the service provider's profit and the owner's total costs to make maintenance contracts more effective. This can be achieved through the development of mathematical models for understanding future costs to be built into the contract price. 
Failure to do so may result in loss to the service provider or the user/owner because of uncertainties associated with system failures and their implication on business.

Estimation of costs for such contracts is complex and it is important to both the owner and the service provider for economic viability. These costs depend on the reliability of the asset/equipment and the maintenance strategies (e.g. corrective maintenance, planned preventive maintenance, and/or inspection procedures) to be considered during the contract period. Maintenance strategy can be developed by understanding the reliability. Failure data are in many cases time or usage dependent for certain conditions. In a probabilistic sense, asset/system failures are functions of usage and/or age.

Only a few cost models for maintenance contracts have been proposed by academic researchers in recent years. Blischke and Murthy ${ }^{2}$ proposed a policy for service contracts with scope for negotiation. Murthy and Yeung ${ }^{5}$ proposed stochastic models for expected profit. Murthy and Ashgarizadeh ${ }^{6}$ developed a model to characterize the optimal strategies for a single customer and single service provider. Ashgarizadeh and Murthy ${ }^{1}$ extended this to multiple customers. Rinsaka and Sandoh ${ }^{9}$ proposed a mathematical model for setting suitable charge of service contract in the case where a manufacturer offers an additional warranty service under which the failed system is replaced by a new one for its first failure, but minimal repairs are carried out to the system for its succeeding failures before the contract expires. $\mathrm{Wu}^{11}$ proposed a maintenance contract model which considered in-house corrective maintenance and outsourced preventive maintenance

Unfortunately, most of these models considered either corrective maintenance (CM) only (rectification only on failure) or preventive maintenance only as maintenance strategy. This type of contract can be dangerous in cases such as maintenance contracts for the rail industry. If a derailment occurs due to rail break/failure as a result of lack of planned maintenance, it will not only cause a loss of billion dollars but it will also cause loss of valuable lives or serious injuries to survivors. Inclusion of both corrective and preventive maintenance in maintenance strategies may prevent this type of accidents in most of the cases, since an on time corrective action as well as a planned preventive maintenance can prolong the reliability of the asset/equipment through proper inspection and on - time maintenance. Therefore, for real life situations, servicing strategies for repairable systems should involve both corrective maintenance (CM) and planned preventive maintenance (PM). This inspires the authors to develop a new maintenance contract model that takes into account both CM and PM.

In this paper, a conceptual cost model for repairable complex asset/equipment is developed to estimate the costs for maintenance contracts taking into account both corrective and planned preventive maintenance as service strategies over the contract period. The developed model is then demonstrated using rail failure data to estimate the costs for maintenance contract for Rail asset.

The outline of this paper is: in Section 1, an introduction of maintenance contract is provided. Section 2 discusses different potential servicing strategies to be considered during the contract period. In section 3, a cost model for maintenance contracts is formulated. In Section 4, the developed model is illustrated with a numerical example. In 
the final section, a summary and contribution of this paper, and scope for future work are discussed.

\section{Maintenance Strategies}

Maintenance strategies for repairable systems involve both corrective maintenance (CM) and planned preventive maintenance (PM). Corrective maintenances are unscheduled actions intended to restore the system/asset to its operational state through corrective actions after occurrence of failures (defects). In contrast, planned preventive maintenance actions are carried out to reduce the likelihood of failures or to prolong the reliability of the asset/equipment and/or to reduce the risk of failures.

Both CM and PM take into account different types of servicing actions which can be used based on the failure mode and type. These actions are classified as per degree of restorability as shown in Figure 1.

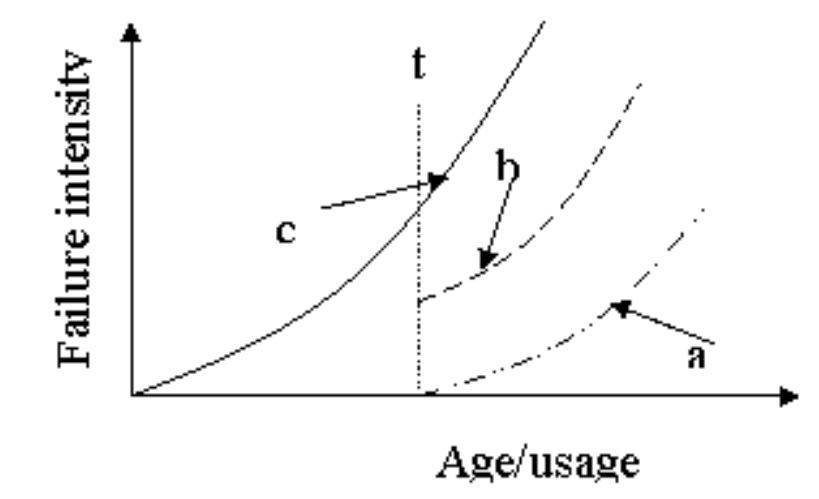

Fig 1: Failure rate with effect of various maintenance actions ${ }^{4}$.

The asset/equipment is considered to fail at point $t$ due to the malfunctioning of one or more components. And various servicing actions can be adopted at this point to restore the functionality of the asset/equipment. The probable servicing strategies applicable for maintenance are:

(i) Replacement: the failed system can be replaced with a new identical system or with a used but good one. This turns failure rate of the item to zero if replaced with new one (see curve 'a' in Figure 1). This implies that a replacement with new and identical system restores the full reliability and turned failure rate to zero. If replaced with used, good system, it restores the part reliability and the failure rate falls to any point between 'as good as new' and 'as bad as old' depending on the age and usage condition of the replaced item/system.

(ii) Overhauling or perfect repair is a restorative maintenance action that enables the system to be "as good as new" condition as it turns failure rate close to zero (See curve 'a' or close to curve 'a'). 
(iii) Imperfect repair restores a substantial portion and like replacement with used, good item, the failure rate falls in between "as good as new" and "as bad as old (see curve ' $b$ ') depending on the type and quality of the repair works.

(iv) A minimal repair is the repair/replacement of only the failed component/s and other components of the item/system remain untouched. This makes insignificant improvement of reliability and the condition after maintenance is called "as bad as old" (curve 'c' in), since the failure rate of other components remain unchanged ${ }^{3}$.

\section{Modeling Cost of Maintenance Contract}

This Section demonstrates the development of a conceptual cost model considering a simple maintenance contract policy in which, the contract terminates when contract period reaches a time or usage level $L$. The contract includes provision for corrective maintenance - rectification on failure as well as constant interval preventive maintenance actions to prolong the system reliability. This can be presented graphically by the Fig 2 . Preventive maintenance actions are carried out at constant interval $x$, each PM restores the reliability of the asset to some extent. Between two successive preventive maintenances there could be one or more imperfect corrective actions. The following assumptions are made for model simplification purpose

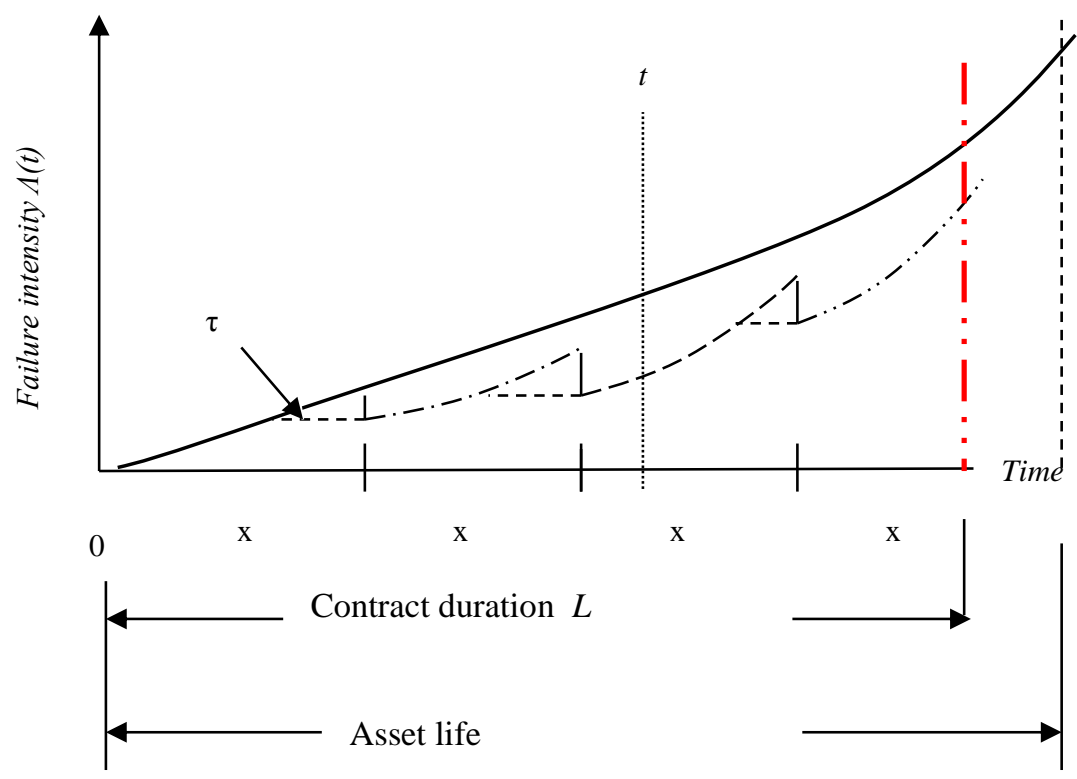

Fig. 2. Graphical representation of the service contract Policy model 1

\subsection{Estimating Maintenance Cost $\left(C_{m}\right)$}

Expected total cost of maintenance service

$=($ Expected total cost of all imperfect corrective repairs over the contract

+ Expected cost of preventive maintenances over the contract $L) /(1+r)^{\mathrm{n}}$ 
Where, $r$ is the discount rate over the period $n$ and $n=1,2,3$,

Expected cost of imperfect repair

$$
C_{m r} \sum_{k=0}^{N+1} \int_{k x}^{(k+1) x} \Lambda(t-k \tau) d t
$$

Expected cost of preventive maintenance during the contract

$$
N C_{p m}
$$

The total expected maintenance cost $C_{m}$ can therefore be expressed as

$$
C_{m}=\left[C_{m r} \sum_{k=0}^{N+1} \int_{k x}^{(k+1) x} \Lambda(t-k \tau) d t+N C_{p m}\right] /(1+r)^{n}
$$

Where, failure intensity $\Lambda_{p m}(t)=\Lambda(t-k \tau)$

$\Lambda_{p m}(t)$ : failure rate at time $t$, with maintenance.

$\Lambda(t)$ : original failure rate at time $t$ when no maintenance is performed.

$N$ : number of times maintenance is performed (including replacement).

$x$ : $\quad$ fixed time interval of maintenance.

$k: \quad k t h \mathrm{PM}, k=1,2,3, \ldots \ldots \ldots$

$\tau$ : $\quad$ restoration out of maintenance action. $\tau=\alpha x$ here, $\alpha$ is the quality of the maintenance

\subsection{Estimating Inspection Cost $\left(C_{i}\right)$}

Total inspection cost $\left(C_{i}\right)$ over the contract can be given by

$$
C_{i}=\left\{\sum_{j=0}^{N_{i}} c_{i} /\left(1+r_{i}\right)^{j}\right\} \times r /\left\{1-\left(\frac{1}{(1+r)^{n}}\right)\right\}
$$

Where,

$$
N_{i}=\operatorname{Integer}\left[\frac{L}{I_{f}}\right]
$$

$N_{i}$ is the expected number of inspection during the contract, and $r_{i}$ is the discounting rate associated with inspection interval. $I_{f}$ is the inspection interval and $r$ is the annual discount rate.

\subsection{Estimating Risk Cost $\left(C_{i}\right)$}

The risk cost associated with system failure and accident is based on the probability of inspection detecting potential failures and failures not being detected by inspection, accident and associated costs. This can be expressed as 


$$
C_{R}=\frac{\sum_{n=0}^{L} E\left[N\left(t_{n}, t_{n+1}\right)\right] *\left[P_{n}(B) * b+\left(1-P_{n}(B)\right) *\left(P_{n}(A) * a\right]\right.}{(1+r)^{n}}
$$

Where,

$a$ is the expected cost per accident;

$b$ is the expected cost of repairing potential failure based on non-destructive test (NDT)

$P_{n}(B)$ is the probability of detecting potential failure using NDT,

$P_{n}(A)$ is the probability of undetected potential failure leading to accident during the interval between $n$th and $(n+1)$ th periods

$E\left[N\left(t_{n}, t_{n+1}\right)\right]$ is the expected number of failure can caused accidents over the interval of $n t h$ and $n+1$ th period. The expression for $E\left[N\left(t_{n}, t_{n}+1\right)\right]$ depends on the accident related failure data of a particular asset and it is different for different asset. So one can do it when they have a set of good accident related data for a particular asset

\subsection{Estimating Total Cost of the Maintenance Contract $\left(C_{T}\right)$}

Therefore, the expected total cost of contract can be obtained by adding all the above costs. The service providers can charge a premium for such service by adding a profit with the total cost of servicing divided by the contract period (number of years/months or usage in thousands hours or Million gross tonnes). This can be expressed by

Total cost of maintenance contract:

$$
\begin{aligned}
& \mathrm{C}_{T}= \\
& {\left[C_{m r}\left(\sum_{k=0}^{N+1} \int_{k x}^{(k+1) x} \Lambda(t-k \tau) d t\right)+C_{p m} N_{i}\right] /(1+r)^{n}} \\
& \quad+\left\{\sum_{j=1}^{N_{i}} c_{i} /\left(1+r_{i}\right)^{j}\right\} \times r /\left\{1-\left(\frac{1}{(1+r)^{n}}\right)\right\}+ \\
& \frac{\sum_{n=0}^{N} E\left[N\left(t_{n+1}, t_{n}\right)\right] *\left[P_{n}(B) * b+\left(1-P_{n}(B)\right) *\left(P_{n}(A) * a\right]\right.}{(1+r)^{n}} \\
& +p
\end{aligned}
$$

Service provider's premium charge per unit time can be expressed by

$$
P_{c}=\frac{C_{T}+\pi}{L}
$$

where, $L$ is the contract period of the Asset and $\pi$ is the total profit marked up by the service provider. These equations can be solved by using Mathematical softwares such as MATLAB, MAPLE when data is available. 


\section{Numerical Example and Analysis of the Model}

This Section demonstrates a Rail track maintenance contract policy where the contract terminates when contract period reaches a usage level ' $L$ ' Millions of Gross Tonnes (MGT). The contract includes provision for corrective maintenance - rectification on failure as well as constant interval preventive maintenance actions to prolong the rail reliability. The proposed maintenance model is slightly modified since the rail track failure mostly depends on usage level (million Gross Tonnes) instead of time and presented graphically with the Fig 3. Preventive maintenance actions are carried out at constant interval ' $x$ ' $M G T$, each PM restores the reliability of the asset to some extent. Between two successive preventive maintenances there could be one or more minimal corrective actions. Rail failures is modeled here as a point process with an intensity function $\Lambda(m)$, where $m$ represents Millions of Gross Tonnes (MGT) of usages and $\Lambda(m)$ is an increasing function of $m$ indicating that the number of failures in a statistical sense increases with MGT. Then, the failure intensity function $\Lambda(m)$ can be derived as

$$
\Lambda(m)=f(m) /\left(1-F(m)=\lambda \beta(\lambda m)^{\beta-1}\right.
$$

Rail track is normally made operational through repair or rectification of the failed segment and no action is taken with regards to the remaining length of the rail in case of detected defects and rail breaks. Since the length of failed segment replaced at each failure is very small relative to the whole track, the rectification action results in a negligible impact on the failure rate of the track as a whole ${ }^{3}$. For the purpose of simplification of this study, we ignore the time value of money (i.e. discount rate $r=0$ ) as the rail failure mainly depends on usage (MGT) not time (Assumption).

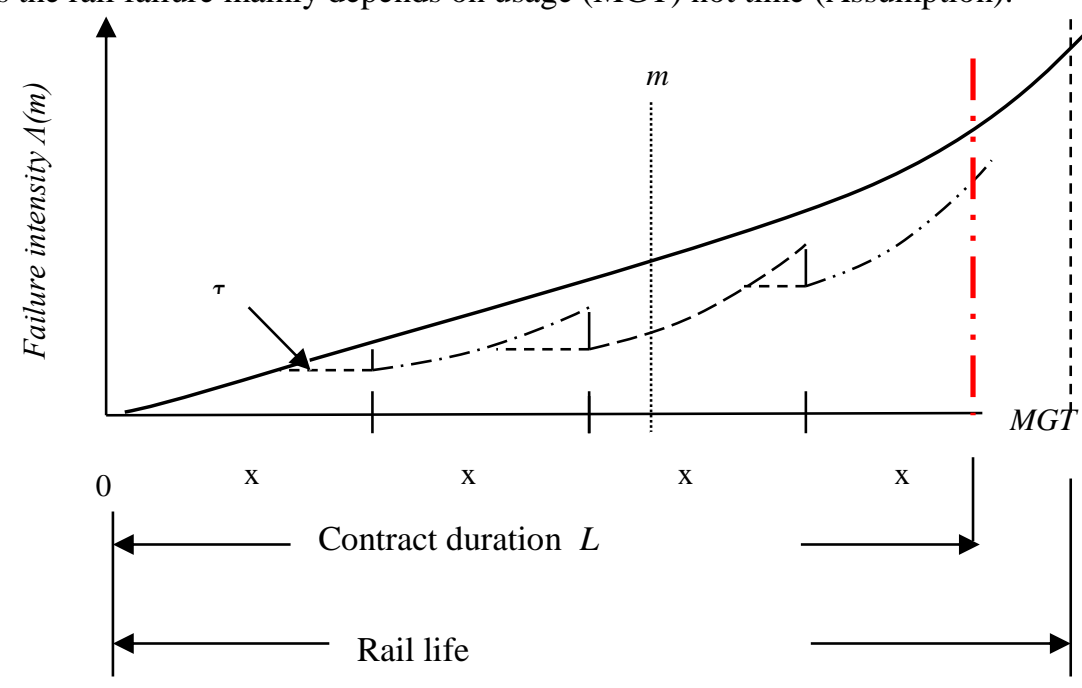

Fig 2: Graphical representation of the service contract Policy model 1

For the purpose of estimating and analyzing the models we used a set of real life rail failure data collected from the Heavy Haul Rail Network. Crude data were first censored 
and rectified to make it useable. The failure or breakage MGT in the analysis is generated as follows: Usage span is considered as 720 MGT. A plot of the accumulated number of rail break versus the accumulated breakage MGT is displayed in the fig 4 .

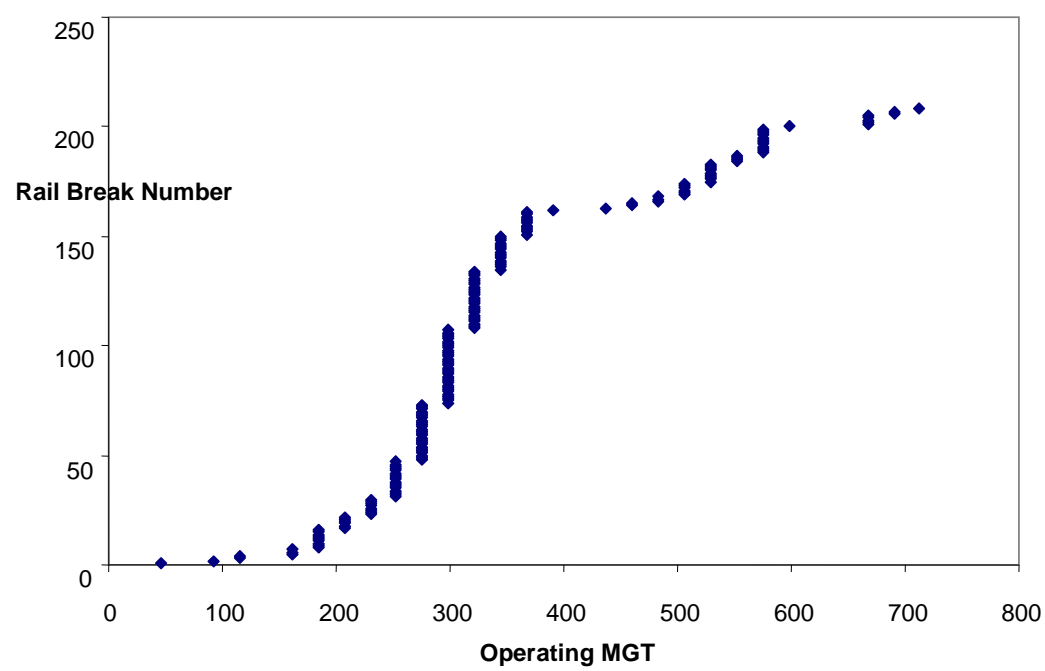

Fig 4: Cumulated Rail break vs. accumulated MGT.

The linearly increasing failure (plotted break) data is an indication that the rate of rail break is not constant. Rather it is usage dependent. Increase of rail breaks with the increase of usage in terms of MGT implies the rail break or failure follows a Non homogeneous Poisson process since the accepted formats of the NHPP are monotone increasing/decreasing functions (Coetzee, 1997).Estimation of Failure parameters In estimating the rail failure parameter, one can use different method such as method of Least square, method of Moments, regression analysis, and method of Maximum likelihood. The method of Maximum Likelihood (MLE) was used here to estimate the parameters $\lambda$ and $\beta$. Parameters were estimated by considering a Weibull distribution (two parameters) by developing a MATLAB program. The MATLAB expression generated inverse characteristic life parameter $\lambda=0.00259$ per MGT and the shape parameter $\beta=2.789$.

\subsection{Estimation Cost of Rail maintenance}

In this section estimated parameters were used in determining the cost of maintenance contract. It is assumed that in each Preventive maintenance action only one pass of grinding and lubrication takes place.

Let us assume for illustration, cost of each minimal repair, cost of replacement/repair of one rail for any Segment due to worn out regulation $C_{m r}=\$ 150$ (Approx)

Cost of each preventive maintenance (rail grinding and Lubrication), $C_{p m}=(\$ 4.00$ per meter (approximately) $\times 110 \mathrm{~m}=\$ 440$. 
Cost of replacement, $C_{r e}=\$ 1700$

Quality of each PM, $\alpha=0.16$, which implies that each PM restores only $16 \%$ of total reliability (we assume it is constant for each PM)

Let the contracted usage in MGT, $L=300$ MGT.

Here, a MAPLE program has been used to determine the optimal interval and number of PMs. This provided the following results

Optimal interval between preventive maintenance $x^{*}=52.65$ MGT

Optimal number of PMs $\mathrm{N}^{*}=5$

Expected total cost of maintenance, $\mathrm{C}_{\mathrm{m}}=$ AUD 1794

\subsection{Expected Inspection Cost}

Let the mean inspection cost over the contract period be $\$ 150$ (includes cost of instruments and inspectors' wage)

Inspection interval over the contract period 30 MGT (Assumed based on historical data)

Total expected Inspection cost, $\mathrm{C}_{\mathrm{i}}=\$ 1500$ throughout the contract period

\subsection{Expected Risk Cost}

Let Mean cost per accident, $a=\$ 10 m$;

Mean cost of repairing potential failure based on NDT, $b=\$ 350$

Probability of detecting potential failure using NDT, $P_{n}(B)=95 \%$

Probability of undetected potential failure leading to accident during contract periods, $\mathrm{P}_{\mathrm{n}}(\mathrm{A})=10^{-7} \%$

$\mathrm{E}[\mathrm{N}(\mathrm{L})]$ is the expected number of failure over the contract period $=5$

Expected risk cost associated with accident, $\mathrm{C}_{\mathrm{R}}=\$ 332.7$.

\subsection{Expected penalty Cost}

Here it assumed a 0 penalty cost (implies for perfect contractor dealings)

\subsection{Total Cost of Maintenance Contract}

Therefore, the total expected cost of maintenance contract $\left(C_{T}\right)$ for a single rail (110metre long) over the 300 MGT usages is estimated as \$3627.

\section{Conclusion}

Maintenance contracts are generally the most economical method for servicing or maintaining highly technical, scientific or complex asset/equipment because of the nature of the asset/equipment and the need to keep its downtime to a minimum. Complex and expensive asset/equipment is normally best maintained or serviced by the original equipment manufacturer (OEM) or its authorized service organization or the third parties interested in investing in infrastructure. Estimation of costs for maintenance contracts is a complex process and is important for both the owners and the service providers. Sevice 
providers need to know the estimated cost to avoid making loss. The owners/ users need to know it to decide contract price so that they are not paying too much compared to the cost of doing that in-house.

In this paper, conceptual cost models for maintenance contract and the service provider's premium charge for complex repairable item are proposed which takes into account both corrective maintenance in the form of minimal repairs and planned preventive maintenances as servicing strategies throughout the contract period. This model also considered accidental risk costs associated with the asset/equipment during the contract period, and penalty provisions for the service provider in case failing to maintain it on time. This model can be applicable to outsourcing maintenance service for any complex repairable systems. This developed conceptual model is then illustrated with a numerical example for rail track maintenance purpose using real life rail data. These models can be further extended by including provisions for used items, and utility functions for linking owner/agent's risk preferences and more complex models could be developed linking downtime.

\section{References}

1. E. Ashgarizadeh and D.N.P. Murthy, Service contract: a stochastic model, Mathematical and Computer Modelling, 31(2000), 11-20.

2. W. R. Blischke, and D.N.P. Murthy, Warranty and service contracts, (John Willey \& Sons Inc. New York, 2000).

3. R. E. Barlow, and L. Hunter, Optimum preventive maintenance policies, Operations Research, 8 (1960), 90-100.

4. Chattopadhyay, G.N. and Rahman, A., Development of lifetime warranty policies and models for estimating costs, Reliability Engineering and System Safety, 93(4), (2008), 522-529.

5. J. L. Coetzee, The role of NHPP models in the practical analysis of maintenance failure data. Reliability Engineering and System Safety, 56, (1997), 161-168.

6. D.N.P. Murthy, and Yeung, Modelling and analysis of service contracts, Mathematical and Computer Modelling, 22(1995), 219-225.

7. D.N.P. Murthy, and E. Ashgarizadeh, "Modelling service contracts" Presented at the INFORMS Meeting in New Orleans, USA, (1995),

8. A. Rahman, and G.N. Chattopadhyay, Modelling cost of maintenance contract for rail infrastructure, in Proc. Int, Conf. of Industrial Engineering and Operations management,Dhaka, Bangladesh, 2010, pp

9. A. Rahman, and G.N. Chattopadhyay, Optimal service contract policies for outsourcing maintenance service of asset to the service provider", International Journal of Reliability and Application, 8(2), (2007), 183-197

10. K. Rinsaka, and H. Sandoh, A stochastic model on an additional warranty service contract, Computers and Mathematics with Applications, 51(2006), 179-188.

11. H. Tarakci, K. Tang, and S. Teyarachakul, "Learning effects on maintenance outsourcing", European Journal of Operational Research, 192 (1), (2007), 449-453.

12. Shaomin $\mathrm{Wu}$, Assessing maintenance contracts when preventive maintenance is outsourced, Reliability Engineering and System Safety, 98(2), (2012), 66-72 
Dr. Anisur Rahman is a Senior lecturer in Engineering Management in Griffith School of Engineering, at Griffith University, Australia. He received his $\mathrm{PhD}$ and Master of Engineering (Thesis) degree in Reliability and maintenance engineering from Queensland University of Technology, Brisbane, Australia in 2007 and 2003 respectively. He obtained a BSc Engineering (Mechanical) degree from Bangladesh University of Engineering Technology, Dhaka. He has been working as University academic since 2001. His research has focused on Operation research, mathematical modelling of product reliability and warranty, asset maintenance and service contract, and climate change and renewable energy. He has published more than 45 articles in various international refereed Journals and international conference proceedings. He is a regular reviewer of Reliability engineering and system safety, IEEE TEM, IIE Transactions and APJOR Journals and many international conferences. 